\title{
Ocular changes in metastatic melanoma patients treated with MEK inhibitor cobimetinib and BRAF inhibitor vemurafenib
}

\author{
Ana Ursula Gavric ${ }^{1}$, Janja Ocvirk ${ }^{2,3}$, Polona Jaki Mekjavic ${ }^{1,3}$ \\ ${ }^{1}$ Eye Hospital, University Medical Centre Ljubljana, Ljubljana, Slovenia \\ ${ }^{2}$ Department of Medical Oncology, Institute of Oncology Ljubljana, Ljubljana, Slovenia \\ ${ }^{3}$ Faculty of Medicine, University of Ljubljana, Ljubljana, Slovenia
}

Radiol Oncol 2018; 52(2): 213-219.

Received 4 October 2017

Accepted 20 November 2017

Correspondence to: Assoc. Prof. Polona Jaki Mekjavić, M.D., Ph.D.; Eye Hospital, University Medical Centre Ljubljana, Grablovičeva 46, 1000 Ljubljana. Phone: +386 1522 1750; Fax: +386 1522 1940; E-mail: polona.jaki@guest.arnes.si

Disclosure: No potential conflicts of interest were disclosed.

Background. Mitogen-activated protein kinase kinase (MEK) inhibitor cobimetinib and V-raf murine sarcoma viral oncogene homolog BI (BRAF) inhibitor vemurafenib have significantly improved the prognosis of BRAF-mutated metastatic melanoma. Some ocular symptoms and signs were recently recognized to follow this treatment. The study was aimed to investigate ocular toxicity in patients with metastatic melanoma treated with cobimetinib in combination with vemurafenib.

Patients and methods. In the prospective, observational study, patients with BRAF-mutated metastatic melanoma treated with cobimetinib in combination with vemurafenib at the Institute of Oncology Ljubljana were asked to participate. Ophthalmic examination was performed including measurement of visual acuity and intraocular pressure, slit lamp examination, funduscopy (CF), infrared-reflectance (IR) imaging and optical coherence tomography (OCT). Results. Five out of 7 patients noticed changes in vision few days after starting the therapy with cobimetinib. In all patients, small circular lesions, described as MEKAR lesions, were documented in outer retinal layers demonstrated with OCT, IR, and CF. Changes were in the center and/or scattered over the retina almost symmetrical in both eyes in 6 patients, and asymmetrical in one patient, the latter presented also with unilateral anterior uveitis and cystoid macular edema.

Conclusions. Multiple bilateral foveal and extrafoveal small retinal lesions in the outer retinal layers develop in patients treated with MEK inhibitor in combination with BRAF inhibitor. Ophthalmologists and oncologists need to be aware of this common, yet relatively benign and often transient ocular side effect to avoid needless intervention, including the discontinuance of a potentially life-prolonging therapy.

Key words: metastatic malignant melanoma; eye; MEKAR; MEK inhibitor

\section{Introduction}

Malignant melanoma is a significant health problem, the incidence of melanoma is steadily increasing, fastest among all cancers. ${ }^{1}$ At diagnosis, metastases are present in approximately $2-5 \%$ of patients. $^{2}$

In recent years several new targeted drugs and immunotherapy have been approved for the treatment of metastatic melanoma. Mitogen-activated protein kinase kinase (MEK) inhibitors and V-raf murine sarcoma viral oncogene homolog B1 (BRAF) inhibitors are a newer group of drugs that act on the target enzymes of MAPK / ERK signaling pathway. The combination treatment with BRAF and MEK inhibitors is amongst the current standard of care for stage IIIC/IV BRAF-mutated melanoma. Combined BRAF and MEK inhibition seems to provide a greater benefit than BRAF or MEK inhibitor monotherapy.3.4 
The BRAF protein is a key part of "mitogen-activated protein kinase signaling pathway" (MAPK), which regulates the division and proliferation of cells and plays a central role in the progression of melanoma. MEK inhibitors inhibit the mitogen-activated protein kinase kinase MEK1 and/or MEK2. A specific mutation in the BRAF gene causes excessive activity, leading to uncontrolled growth and cell division. About $50 \%$ of melanomas harbors activating BRAF mutations. ${ }^{5}$ Therapy with the selective inhibitor of mutant BRAF Val600, vemurafenib in combination with MEK inhibitor cobimetinib showed major tumor responses, resulting in improved progression-free and overall survival in patients with metastatic disease, compared with chemotherapy. 3,6

MEK inhibitors have been associated with changes in retina by means of small localized serous neuroretinal detachments, which have been named MEK inhibitor-associated retinopathy (MEKAR). ${ }^{7,8}$ The most common ocular side effect associated with BRAF inhibitor vemurafenib in monotherapy are uveitis, conjunctivitis and dry eyes. ${ }^{9}$

When used cobimetinib in combination with vemurafenib the most commonly observed adverse effects in the coBRIM phase III trial were rash, photosensitivity, MEKAR, alopecia, hyperkeratosis, diarrhea, nausea, arthralgia, fatigue, vomiting, blood creatine phosphokinase (CPK) increase and alanine aminotransferase (ALT) increase. Most adverse effects occurred within the first treatment cycle and decrease substantially over time. ${ }^{10}$

A pattern of ocular toxicity has followed these drugs through clinical trials and their association with ocular toxicity is only just beginning to be recognized.

The aim of our study was to look prospectively at the incidence, symptomatology, course, and reversibility of ocular toxicity in patients with BRAFV600 metastatic melanoma treated with MEK/BRAF inhibitor cobimetinib plus vemurafenib.

\section{Patients and methods}

\section{Patients}

All patients with BRAFV600 metastatic melanoma treated at the Institute of Oncology Ljubljana with MEK/BRAF inhibitor cobimetinib and vemurafenib from January to June 2017, were offered the chance to be included in the study at Eye Hospital of University Medical Centre Ljubljana. Seven out of eight patients were willing to participate. The

TABLE 1. Patient characteristics

\begin{tabular}{|c|c|c|c|c|c|c|c|}
\hline Patient $\mathbf{N}^{\circ}$ & 1 & 2 & 3 & 4 & 5 & 6 & 7 \\
\hline Sex & $M$ & M & $\mathrm{F}$ & $M$ & $M$ & $M$ & $M$ \\
\hline Age (years) & 41 & 58 & 64 & 74 & 71 & 45 & 55 \\
\hline No of cycles at first eye exam & 2 & 11 & 15 & 3 & 3 & 3 & 6 \\
\hline No of cycles at last eye exam & 3 & 13 & 24 & 8 & 5 & 4 & 7 \\
\hline Change in dosage & yes* & no & no & no & no & no & no \\
\hline BCVA RE & 1.0 & 1.0 & 1.0 & $0.7-0.9$ & 0.8 & 1.0 & 1.0 \\
\hline BCVA LE & 1.0 & 1.0 & 1.0 & $0.7-0.9$ & 0.7 & 1.0 & 1.0 \\
\hline Symptoms & $\begin{array}{l}\text { circle } \\
\text { centrally }\end{array}$ & $\begin{array}{l}\text { blurred } \\
\text { vision }\end{array}$ & no & $\begin{array}{l}\text { blurred } \\
\text { vision }\end{array}$ & $\begin{array}{l}\text { floaters in } L E \text {, } \\
\text { blurred vision }\end{array}$ & $\begin{array}{l}\text { circle } \\
\text { centrally }\end{array}$ & no \\
\hline $\begin{array}{l}\text { Occurrence of symptoms } \\
\text { after starting the therapy }\end{array}$ & 1 week & 1 week & - & 1 week & 1 month & 1 week & - \\
\hline Fluctuations of symptoms & $\begin{array}{l}\text { spontan. } \\
\text { resolution }\end{array}$ & no & - & $\begin{array}{l}\text { different } \\
\text { spectacles } \\
\text { needed }\end{array}$ & $\begin{array}{l}\text { better after } \\
\text { topical therapy }\end{array}$ & $\begin{array}{l}\text { spontan. } \\
\text { resolution }\end{array}$ & needs spectacles \\
\hline OCT changes in the fovea & $\begin{array}{l}\text { elongation } \\
\text { of IZ }\end{array}$ & SRF & SRF & SRF & $\begin{array}{l}\text { elongation of IZ } \\
\text { in RE, SRF in LE }\end{array}$ & SRF & SRF \\
\hline Extrafoveal SRF & $\begin{array}{l}\text { multiple } \\
\text { bilateral }\end{array}$ & $\begin{array}{l}\text { multiple } \\
\text { bilateral }\end{array}$ & $\begin{array}{l}\text { multiple } \\
\text { bilateral }\end{array}$ & $\begin{array}{l}\text { multiple } \\
\text { bilateral }\end{array}$ & no & no & multiple bilateral \\
\hline Other ocular findings & - & - & - & $\begin{array}{l}\text { incipient } \\
\text { senile } \\
\text { cataract ou }\end{array}$ & $\begin{array}{l}\text { uveitis in LE; } \\
\text { incipient } \\
\text { cataract ou }\end{array}$ & - & $\begin{array}{l}\text { dilatated } \\
\text { conjuctival } \\
\text { vessels in the RE }\end{array}$ \\
\hline
\end{tabular}

* adjusted dose = lower dose due to the cutaneous side effects; $B C V A=$ best corrected visual acuity; $F=$ female; $I Z=$ interdigitation zone; $L E=l$ left eye; $M=$ male, $O C T=$ optical coherence tomography; ou = both eyes; RE = right eye; SRF = subretinal fluid 
study adhered to the tenets of the Declaration of Helsinki and was submitted and approved by The Slovenian National Medical Ethics Committee. All patients signed an informed consent form and all attended the ophthalmology visits.

\section{Treatment}

All patients received BRAF/MEK inhibitor combination therapy for metastatic melanoma, vemurafenib (Zelboraf, Roche Products Ltd, Hertfordshire, UK) plus cobimetinib (Cotellic, Roche Products Ltd, Hertfordshire, UK) in the standard doses: cobimetinib $60 \mathrm{mg}$ orally once daily on days 1-21 of an every 28-day cycle and vemurafenib $960 \mathrm{mg}$ orally twice daily on days 1-28. Doses were reduced in the course of the treatment in one patient due to a severe cutaneous side effect.

\section{Outcome measures}

All patients had undergone a complete ophthalmologic examination, including best-corrected visual acuity (BCVA) and intraocular pressure measurement, slit-lamp biomicroscopy, funduscopy, and noninvasive imaging: color fundus photography (Topcon Fundus Camera), fundus autofluorescence (FAF), infrared-reflectance (IR) and spectral-domain optical coherent tomography (SDOCT). Images were obtained with the Heidelberg Spectralis HRA+OCT (Heidelberg Engineering, Heidelberg, Germany). Every patient had at least two examinations in Eye Hospital of University Medical Centre Ljubljana.

Information about primary cancer diagnosis, other adjuvant treatments, the number of cycles, and the dosage of chemotherapy was collected from medical charts.

\section{Results}

\section{Patient characteristics}

There were 7 participants with BRAF mutated metastatic melanoma. The mean age was 58.3 years $( \pm$ 9.8 years) with a median age of 58 years (range of 41-74 years). Time from the beginning of the therapy to the last control ranged from 2 to 24 months. Patient characteristics are shown in Table 1.

\section{Ophthalmic characteristics}

Ocular adverse effects were documented in all of the patients, giving an incidence of $100 \%$. Bilateral
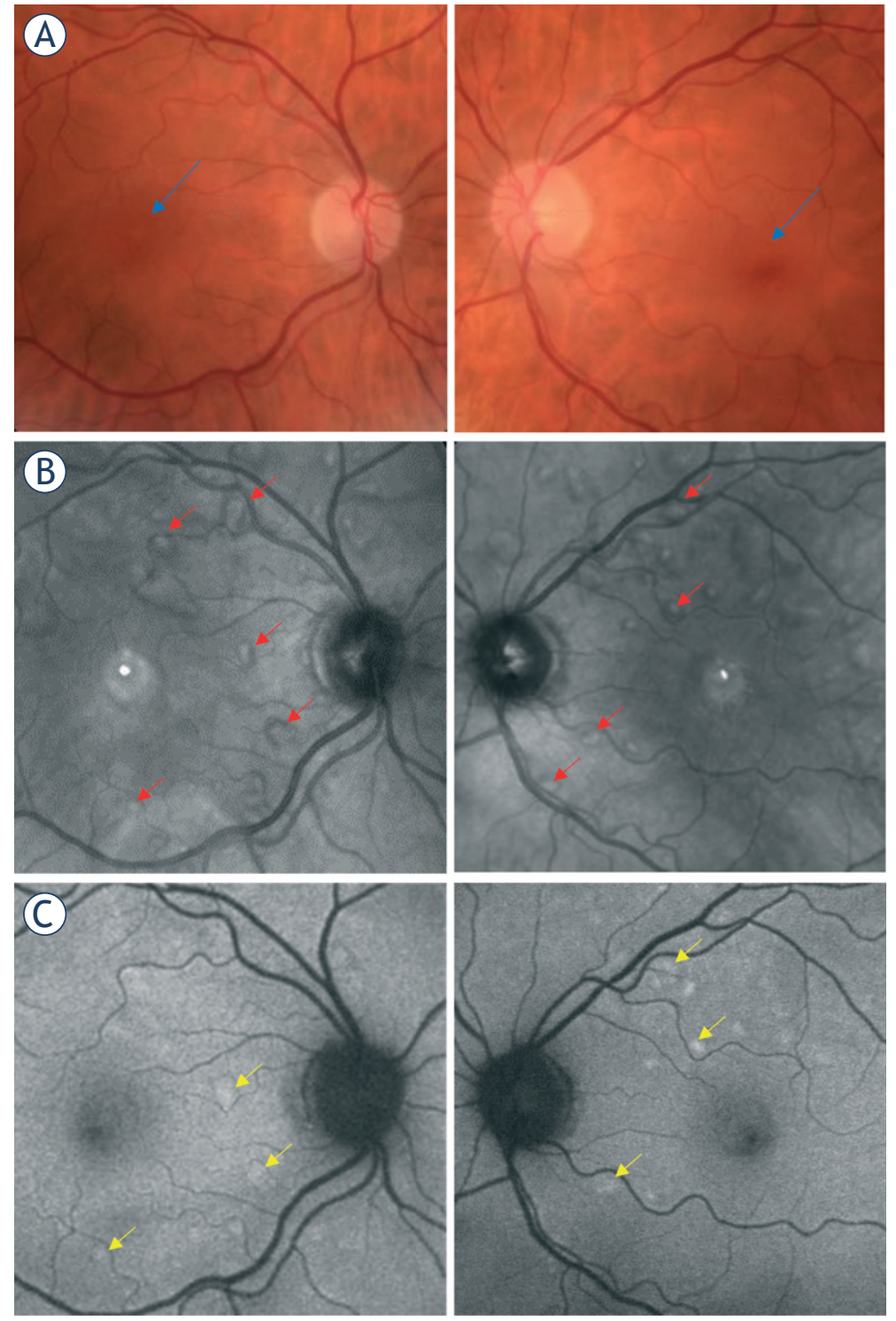

FIGURE 1. (A) Discrete bilateral lesions in the fovea seen on fundus photography (blue arrows); (B) Infrared reflectance imaging showing lesions with a hyperreflective center, surrounded by a hyporeflective zone scattered throughout the posterior pole (some are marked with red arrows); (C) Some lesions showed increased signal in autofluorescence imaging (some are marked with yellow arrows).

serous neuroretinal detachments were observed in all 7 patients, additionally, in 1 eye, there was an associated anterior uveitis with cystoid macular edema (CME).

Five patients reported eye symptoms, 3 had fluctuations of blurry vision and 2 reported a circle centrally in the visual field. Best corrected visual acuity (BCVA) was 1.0 in 5 patients. Two patients had reduced BCVA; one presented with incipient senile cataract, his BCVA fluctuated from of 0.7 to 0.9 in both eyes, another patient presented with unilateral uveitis and cataract in both eyes, he has 


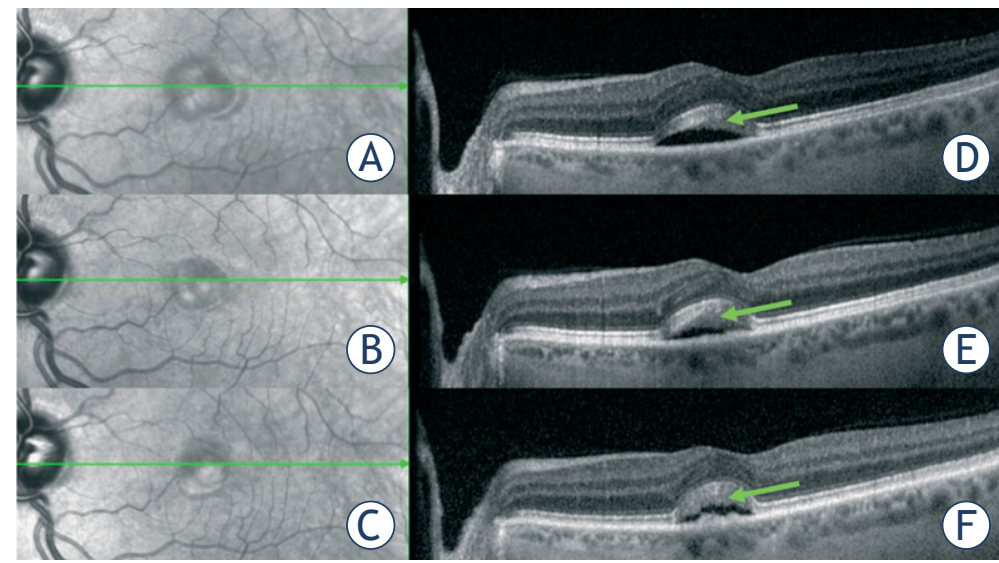

FIGURE 2. (A-C) IR imaging of foveal lesion during cycles; (D-F) OCT showed fluctuations of dome-shaped accumulation of subretinal fluid with elongation of interdigitation zone (green arrows) in the foveal region; $A+D=$ imaging during $3^{\text {rd }}$ cycle; $\mathrm{B}+\mathrm{E}=$ imaging during $6^{\text {th }} \mathrm{Cycle} ; \mathrm{C}+\mathrm{F}=$ imaging during $8^{\text {th }}$ cycle.
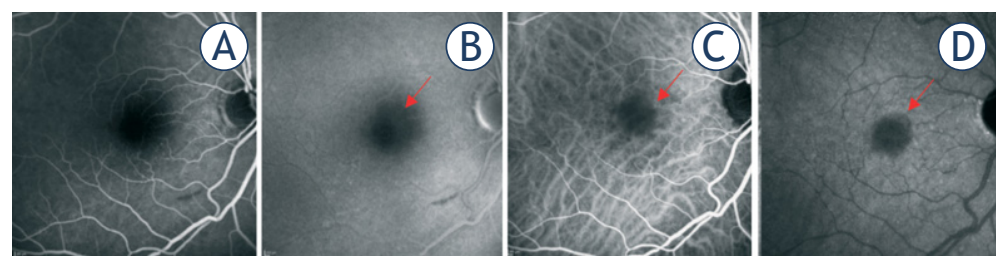

FIGURE 3. Fluorescein (A,B) and indocyanine green (C,D) angiography showed only mild masking effect of subretinal fluid on the location of lesions (red arrows).

BCVA of 0.6. IOP remained stable in all patients (from 14 to $22 \mathrm{mmHg}$ ).

Ophthalmoscopy revealed discrete bilateral transparent to yellowish lesions of less than $1 / 3$ of the disc diameter in the fovea and extrafoveally. Not all lesions were seen with ophthalmoscopy (Figure 1A).

IR imaging showed lesions with a hyper reflective center, surrounded by a hyporeflective zone and multiple smaller lesions with similar reflectance characteristics scattered throughout the posterior pole. The location and number of lesions were relatively symmetrical between both eyes (Figure 1B).

Some lesions showed increased signal in autofluorescence imaging (Figure 1C).

Dome-shaped accumulations of subretinal fluid (SRF) with elongation of outer part of the interdigitation zone of photoreceptors into hyporeflective SRF were detected on OCT. During the following cycles of therapy, OCT findings showed fluctuations (Figure 2).

One patient presented with anterior uveitis and cystoid macular edema in the left eye, which re- solved after a 3-week course of topical $0.1 \%$ dexamethasone.

Besides noninvasive imaging, fluorescein and indocyanine green (ICG) angiography was performed in one patient, showing no vascular abnormalities, no leakage or staining, only mild masking effect of subretinal fluid on the location of lesions was observed (Figure 3).

\section{Discussion}

We studied the incidence, symptoms and clinical characteristics of ocular toxicity in patients with BRAFV600 metastatic melanoma treated with cobimetinib in combination with vemurafenib.

Our results show 100\% incidence of MEKAR in our patients. In contrast, in the coBRIM study patients were receiving the same therapy, but MEKAR was reported in only $26 \%$ of patients. ${ }^{11}$ The authors of this study do not specify the day and hour of the examination, which according to Urner-Bloch is very relevant. On the basis of our observations, we concur with Urner-Bloch that the fluctuations in the MEKAR lesions are also dependent of the day within the treatment cycle. ${ }^{7}$ In patients that were also examined during the inter-treatment interval, we noted a decrease in the magnitude of the lesions. Some patients reported fluctuation of the symptoms, and one of them was able to compensate fluctuated blurry vision with different hyperopic refractive correction.

The results of a meta-analysis of the ocular safety of MEK inhibitors reveals an increased risk for ocular problems, particularly disturbed vision, and retinal changes such as "chorioretinopathy and retinal detachment". They did not report any statistically significant increase in the risk for uveitis or eye hemorrhage. ${ }^{12}$

Similar results to ours were reported in a prospective observational study, in which 62 melanoma patients were treated with MEK inhibitor binimetinib in monotherapy or in combination with BRAF inhibitor. Bilateral retinopathy was diagnosed in $92 \%$ of patients on monotherapy and $100 \%$ in those with combination therapy. Retinopathy was described as dose- and time-dependent and was reversible in all patients. ${ }^{7}$

There is some confusion regarding the description of MEKAR in the literature. Some groups and authors have defined MEKAR as "chorioretinopathy", "retinal pigment epitelium detachement (PED)" or "central serous retinopathy-like". 12-15 
By means of optical coherent tomography (OCT) which became widely used diagnostic tool for retina, the morphology of MEKAR lesions is clarified. Lesions in MEKAR are not PED, because RPE is not detached and the fluid is accumulated above it. It is also not chorioretinopathy, as there are no visible changes in the choroid, no pachycoroid is observed in MEKAR and also no observable changes on ICG angiography. All mentioned changes are typical for central serous chorioretinopathy. ${ }^{8}$

Characteristically, in MEKAR, the choroidal thickness is normal, and focal lesions in the outer retinal layers are notable. The ellipsoid layer is unchanged and is clearly distinguishable from interdigital zone (IZ) which has elongations into sub-IZ space. Sub-IZ is filled with hypo reflective fluid (SRF). RPE layer is not disturbed. During the cycles hypo reflective sub-IZ fluid fluctuates, can vanish, but elongations of IZ remain (Figure 2). Previous studies have described similar retinal lesions associated with other MEK inhibitors treatment., 7,16,17

If lesion is located in the strict center of the macula, in the foveola, patients can describe symptoms such as a circle centrally in the visual field or blurred near vision. Accumulation of SRF thickens retina in the place of lesion hence the axial length of the eye is shortened. That results in blurred near vision due to induced hyperopia. Patients complain of difficulty reading without or with inappropriate reading glasses. Because lesions in MEKAR are thin, changes in vision are not dramatic and do not importantly influence patients' quality of life. If the symptoms are disturbing, they can be corrected with mild hyperopic correction.

One patient noticed a circle centrally in the visual field few days after starting the therapy. The therapy was promptly discontinued due to cutaneous side effects and after cessation of the therapy, the ocular symptoms also disappeared. Two weeks later patient started taking low dose therapy (cobimetinib $40 \mathrm{mg} /$ day for 3 weeks, and vemurafenib $720 \mathrm{mg} / 12 \mathrm{~h}$ ) and the ocular symptoms did not reappear. Morphological changes were present but barely detectable (Figure 4). It has been reported that patients treated with MEK inhibitor biminetinib experience visual disturbances, particularly in the first 4 weeks of treatment. Subsequent treatment, initiated after a given interval, causes less pronounced symptoms. ${ }^{7}$ It has not been reported whether the dosage within a given cycle influences the occurrence of the symptoms or the amount of sub-IZ fluid.

The mechanism of MEK inhibitor ocular toxicity still remains unresolved. Dysfunction of retinal

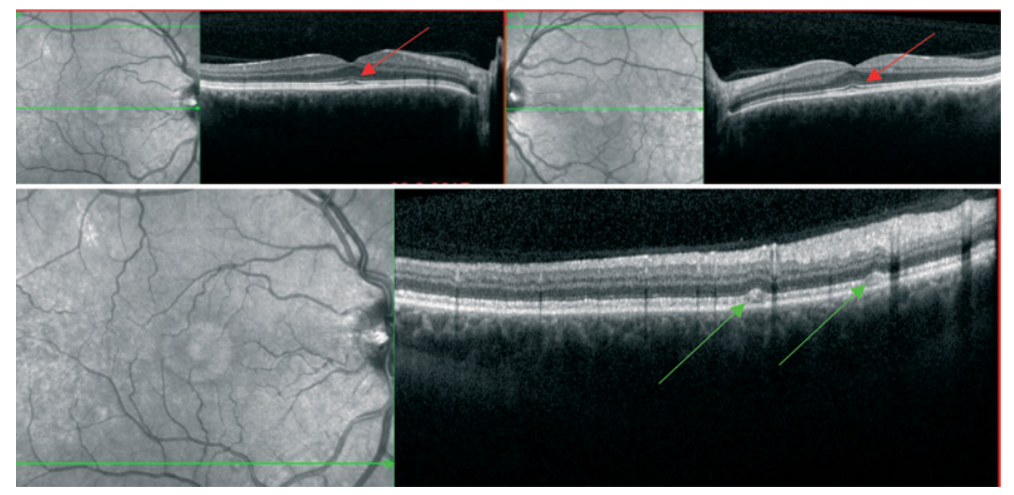

FIGURE 4. Discrete morphological changes with focal elongations of interdgitation zone in the fovea (red arrows) and near the vascular arcades (green arrows) seen on OCT.

pigmented epithelium (RPE) may play a major role in pathogenesis. ${ }^{18}$ Ophthalmoscopy revealed multiple barely noticeable lesions of less than $1 / 3$ of the disc diameter distributed in the posterior part of the both eyes, in the fovea and scattered around it extrafoveally, mainly near the vascular arcades. It is speculated that these are the sites where the concentration of the drug is the highest. ${ }^{8}$ Prior preclinical studies showed that MEK inhibition leads to acute RPE toxicity, which results in RPE hyper permeability and breakdown of the retinal-blood barrier. ${ }^{19,20}$ RPE is an epithelial barrier that maintains the outer blood-retinal barrier. Adherent and tight junctions, facilitated and active transporters perform important barrier functions in the physiological processes of the retina, by transporting nutrients, water, and ions, and removing metabolic wastes. The removal of excessive water from the subretinal space to the choriocapillaris, which is critical to the health of the retina, is regulated by the active transport systems in the RPE. ${ }^{21}$ Inappropriate function of RPE may lead to SRF accumulation observed in MEKAR. Lesions in the foveola are observed to be thicker than extrafoveally. The possible explanation for that might be the fact that in the fovea the concentration of photoreceptors is the greatest, hence this is the region of greatest active transport from the retina to choroid.

OCT reveals some changes in outer retinal layers, observed changes are focal: clearly distinguishable IZ with elongations into sub-IZ space, sub-IZ space can be additionally thickened due to accumulation of hyporeflective SRF above RPE layer which remains unchanged.

During the subsequent treatment, OCT findings showed minimal fluctuations in SRF. In our patients, the follow-up was up to 2 years from the 
beginning of the treatment. During this time, lesions did not completely resolve. Despite persistent SRF, no permanent changes in RPE layer were observed in this short-term follow up. Van Dijk et al. analyzed the molecular mechanism and cell proliferation using neuroretina and cell models of RPE treated with MEK inhibitor binimetinib in vitro and found that cell proliferation was not inhibited during treatment and retinal cells were able to regain the activation after binimetinib treatment, mimicking the reversibility of the retinopathy. ${ }^{16}$ The effect on RPE on long-term follow up is to be studied further.

We also observed discrete focal dilatation of conjunctival vessels by slit lamp microscopy in one patient. These changes were not previously reported in the literature and could be the result of local irritation due to some other factor.

Patient number 5 presented with reduced vision in his left eye. His BCVA was reduced to 0.7, and he observed floaters. We found cells in the anterior chamber with posterior synechiae as well as slight macular edema. After topical corticosteroid therapy, the signs and symptoms resolved. This is in line with previous case reports of patients with metastatic melanoma and clinical signs of inflammation in both eyes. The bilateral uveitis was reported as an adverse effect of vemurafenib therapy, which was treated with topical corticosteroid therapy. ${ }^{22,23}$ In our patient number 5, MEKAR with lesions in both foveas was observed three weeks after the onset of inflammation in the left eye. Previous studies have reported that the most common ocular toxicity associated with BRAF inhibitor monotherapy is uveitis, occurring in $2.1 \%$ of patients receiving vemurafenib and in $1 \%$ of patients treated with dabrafenib across clinical trials. Current algorithms suggest management with a temporary dose interruption, ophthalmological review, a course of topical steroids and in most cases a dose reduction. ${ }^{13}$

We have observed a high incidence of ocular side effects in this small group of patients taking combined cobimetinib and vemurafenib. They were mild and prone to spontaneous resolution. Morphological changes were located in outer retinal layers. They did not significantly effect visual function. Overall, we have shown that therapy with MEK/BRAK inhibitor combination therapy is safe with respect to ocular adverse effects, for a duration of up to 2 years. However, the results of the present study must be viewed as a hypothesisgenerating pilot study, and the results interpreted with caution, because of the small sample sizes.
We would recommend that patients treated with MEK and BRAF inhibitors have regular ophthalmologic examinations, which include BCVA, slit lamp examination, and OCT of macular region, and when available IR imaging. The long-term follow-up of MEK inhibitor therapy is to be studied to evaluate the potential irreversible effects of MEK on outer retinal layers, which could permanently influence visual function.

Retinal changes named MEKAR are observed in metastatic melanoma patients treated with MEK inhibitor in combination with BRAF inhibitor. Lesions are either solitary or multiple bilateral foveal and extrafoveal serous neurosensory retinal detachments. MEKAR lesions can be recognized and differentiated from serous retinal detachments of other etiology using noninvasive diagnostic imaging methods such as OCT and IR imaging. They have no, or only mild influence on visual function and are self-limited.

Ophthalmologists and oncologists need to be aware of this common, yet relatively benign and often transient ocular side effect of treatment with MEK inhibitor in combination with BRAF inhibitor to avoid needless intervention, including the discontinuance of a potentially life-prolonging therapy.

\section{References}

1. Arnold M, Holterhues C, Hollestein LM, Coebergh JWW, Nijsten T, Pukkala $\mathrm{E}$, et al. Trends in incidence and predictions of cutaneous melanoma across Europe up to 2015. J Eur Acad Dermatol Venereol JEADV 2014; 28: 1170-8. doi: $10.1111 / \mathrm{jdv} .12236$

2. Garbe $C$, Peris K, Hauschild A, Saiag P, Middleton M, Bastholt L, et al. Diagnosis and treatment of melanoma. European consensus-based interdisciplinary guideline - Update 2016. Eur J Cancer 2016; 63: 201-17. doi: 10.1016/j.ejca.2016.05.005

3. Larkin J, Ascierto PA, Dréno $B$, Atkinson $V$, Liszkay $G$, Maio $M$, et al. Combined vemurafenib and cobimetinib in BRAF-mutated melanoma. $N$ Engl J Med 2014; 371: 1867-76. doi: 10.1056/NEJMoa1408868

4. Dummer R, Hauschild A, Lindenblatt N, Pentheroudakis G, Keilholz U. Cutaneous melanoma: ESMO Clinical Practice Guidelines for diagnosis, treatment and follow-up. Ann Oncol 2015; 26: v126-32. doi: 10.1093/annonc/mdv297

5. Ascierto PA, Kirkwood JM, Grob J-J, Simeone E, Grimaldi AM, Maio M, et al The role of BRAF V600 mutation in melanoma. J Transl Med 2012; 10: 85. doi: 10.1186/1479-5876-10-85

6. Jang $S$, Atkins MB. Which drug, and when, for patients with BRAFmutant melanoma? Lancet Oncol 2013; 14: e60-9. doi: 10.1016/S14702045(12)70539-9

7. Urner-Bloch $U$, Urner $M$, Jaberg-Bentele $N$, Frauchiger AL, Dummer R, Goldinger SM. MEK inhibitor-associated retinopathy (MEKAR) in metastatic melanoma: Long-term ophthalmic effects. Eur J Cancer 2016; 65: 130-8. doi: 10.1016/j.ejca.2016.06.018

8. Francis JH, Habib LA, Abramson DH, Yannuzzi LA, Heinemann M, Gounder $\mathrm{MM}$, et al. Clinical and morphologic characteristics of MEK inhibitorassociated retinopathy: differences from central serous chorioretinopathy. Ophthalmology 2017. doi:10.1016/j.ophtha.2017.05.038. [Epub ahead of print] 
9. Choe $\mathrm{CH}$, McArthur GA, Caro I, Kempen JH, Amaravadi RK. Ocular toxicity in BRAF mutant cutaneous melanoma patients treated with vemurafenib. Am J Ophthalmol 2014; 158: 831-837.e2. doi: 10.1016/j.ajo.2014.07.003

10. Dréno B, Ribas A, Larkin J, Ascierto PA, Hauschild A, Thomas L, et al. Incidence, course, and management of toxicities associated with cobimetinib in combination with vemurafenib in the coBRIM study. Ann Oncol 2017; 28: 1137-44. doi: 10.1093/annonc/mdx040

11. de la Cruz-Merino L, Di Guardo L, Grob J-J, Venosa A, Larkin J, McArthur GA, et al. Clinical features of serous retinopathy observed with cobimetinib in patients with BRAF-mutated melanoma treated in the randomized coBRIM study. J Transl Med 2017; 15: 146. doi: 10.1186/s12967-017-1246-0

12. Alves C, Ribeiro I, Penedones A, Mendes D, Batel Marques F. Risk of ophthalmic adverse effects in patients treated with MEK inhibitors: A systematic review and meta-analysis. Ophthalmic Res 2017; 57: 60-9. doi: 10.1159/000446845

13. Welsh SJ, Corrie PG. Management of BRAF and MEK inhibitor toxicities in patients with metastatic melanoma. Ther Adv Med Oncol 2015; 7: 122-36. doi: $10.1177 / 1758834014566428$

14. Schoenberger SD, Kim SJ. Bilateral multifocal central serous-like chorioretinopathy due to MEK inhibition for metastatic cutaneous melanoma. Case Rep Ophthalmol Med 2013; 2013: 1-3. doi: 10.1155/2013/673796

15. Michalarea V, de Miguel Luken MJ, Diamantis N, Garg A, Maubon L, Yap $\mathrm{TA}$, et al. Ocular toxicity with MEK inhibitors in phase I trials: A single centre experience across six clinical trials. J Clin Oncol 2015; 33: 11090-90. doi: 10.1200/jco.2015.33.15 suppl.11090

16. van Dijk EHC, van Herpen CML, Marinkovic M, Haanen JBAG, Amundson $D$, Luyten GPM, et al. Serous retinopathy associated with mitogen-activated protein kinase inhibition (Binimetinib) for metastatic cutaneous and uveal melanoma. Ophthalmology 2015; 122: 1907-16. doi: 10.1016/j. ophtha.2015.05.027

17. Weber ML, Liang MC, Flaherty KT, Heier JS. Subretinal fluid associated with MEK inhibitor use in the treatment of systemic cancer. JAMA Ophthalmol 2016; 134: 855-62. doi: 10.1001/jamaophthalmol.2016.0090

18. Stjepanovic N, Velazquez-Martin JP, Bedard PL. Ocular toxicities of MEK inhibitors and other targeted therapies. Ann Oncol 2016; 27: 998-1005. doi: 10.1093/annonc/mdw100

19. Jiang $Q$, Cao $C$, Lu S, Kivlin R, Wallin B, Chu W, et al. MEK/ERK pathway mediates UVB-induced AQP1 downregulation and water permeability impairment in human retinal pigment epithelial cells. Int J Mol Med 2009; 23: $771-7$.

20. Huang W, Yang AH, Matsumoto D, Collette W, Marroquin L, Ko M, et al. PD0325901, a mitogen-activated protein kinase kinase inhibitor, produces ocular toxicity in a rabbit animal model of retinal vein occlusion. $J \mathrm{Ocu}$ Pharmacol 2009; 25: 519-30. doi: 10.1089/jop.2009.0060

21. Runkle EA, Antonetti DA. The blood-retinal barrier: Structure and functional significance. In: Nag S, editor. Blood-Brain Neural Barriers, vol. 686, Totowa, NJ: Humana Press; 2011. p. 133-48.

22. Niro A, Strippoli S, Alessio G, Sborgia L, Recchimurzo N, Guida M. Ocular toxicity in metastatic melanoma patients treated with mitogen-activated protein kinase inhibitors: a case series. Am J Ophthalmol 2015; 160: 959-67. e1. doi: 10.1016/j.ajo.2015.07.035

23. Guedj M, Quéant A, Funck-Brentano E, Kramkimel N, Lellouch J, Monnet $D$, et al. Uveitis in patients with late-stage cutaneous melanoma treated with vemurafenib. JAMA Ophthalmol 2014; 132: 1421-5. doi: 10.1001/ jamaophthalmol.2014.3024 